\title{
Inclusion control and fatigue properties of NiTi wires for medical applications
}

\author{
S. Beretta ${ }^{1, a}$, A. Cadelli ${ }^{2}$, A. Coda ${ }^{2}$, P. Luccarelli ${ }^{1}$ and M. Urbano ${ }^{2}$ \\ ${ }^{1}$ Politecnico di Milano, Dipartimento di Meccanica, 20156 Milano, Italy \\ ${ }^{2}$ SAES Getters S.p.A., 20020 Lainate, Italy
}

\begin{abstract}
Superelastic wires of shape memory alloys are used for medical devices, but there has not been a systematic study about the correlations between fatigue properties and inclusion content. In this study we present a thorough investigation about the fatigue properties of $0.3 \mathrm{~mm}$ nitinol wires subjected to rotating bending fatigue. A comprehensive experimental campaign was conducted involving tests at different strain amplitudes for obtaining fatigue curves. The fracture surfaces of failed wires were analyzed through scanning electron microscopy, and data regarding the presence of particles and their morphology were recorded. We show qualitatively how inclusion dimensions affect fatigue. Finally, by means of extreme value statistics, we rate the particle dimensions of the four processes and correlate them to fatigue performance.
\end{abstract}

\section{Introduction}

Significant efforts have been devoted to the study of the fatigue behavior of nitinol [1, 2]. To our knowledge, however, no systematic research has been performed to study the possible effects of inclusions on the fatigue performance of nitinol, and only a few papers treat this issue. The limited knowledge about the effect of inclusions is reflected in the regulations pertinent to the characterization of inclusions in nitinol (ASTM F2063-05).

In this work, we present the first part of a research program aimed at verifying that the findings of Murakami et al. [3] about the effect of inclusions are applicable to nitinol. We have selected lots of superelastic wires manufactured with differing melting techniques and hot processing steps but presenting almost equal thermo-mechanical properties. The process differences are supposed (this is verified a posteriori) to influence the inclusion size and nature of superelastic wires. Wires underwent RBT at several strain levels, and fracture sections were observed via field emission scanning electron microscopy, for measuring the dimensions of the inclusions present at the fracture nucleation sites.

The whole set of fatigue data collected for each process was analyzed through an interpretative model, namely, the bilinear uniform scatter band model. This analysis allows us to appreciate quantitatively how different processes influence the fatigue behaviors, in particular in the

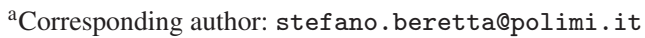

This is an Open Access article distributed under the terms of the Creative Commons Attribution License 4.0, which permits unrestricted use, distribution, and reproduction in any medium, provided the original work is properly cited. 


\section{MATEC Web of Conferences}
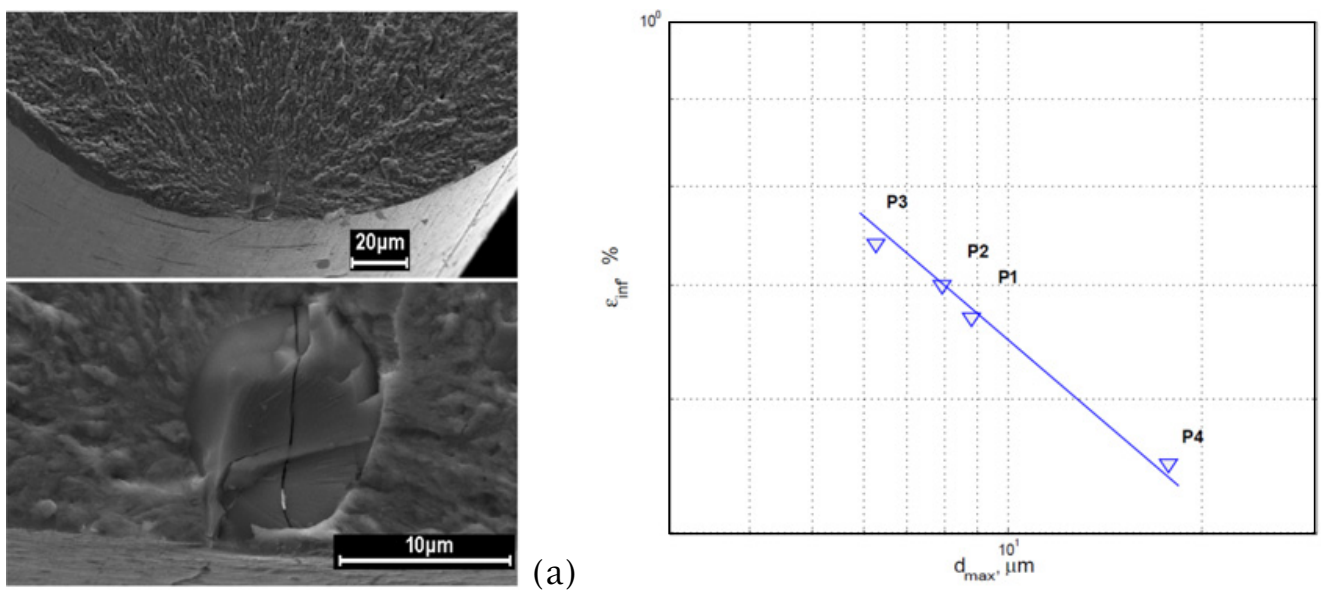

(a)

Figure 1. Fatigue tests on nitinol wires [4]: a) fracture surface showing an inclusion at fracture origin; b) correlation between fatigue limit (expressed in strain) and dimension of extreme inclusions.

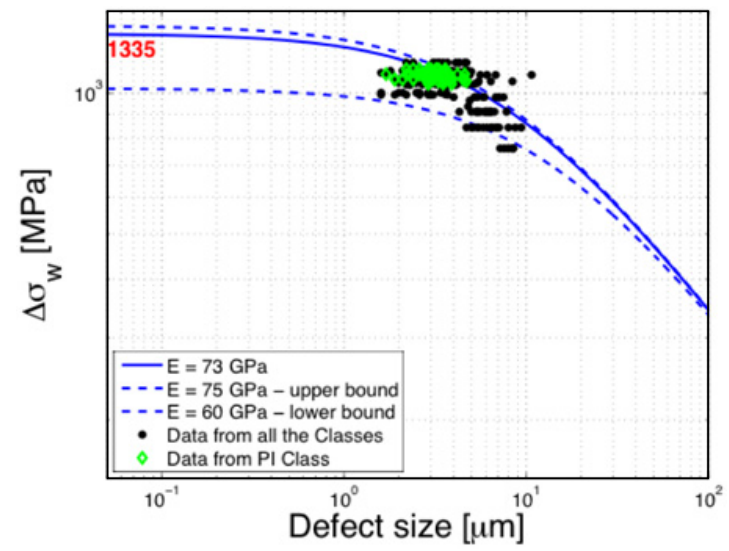

Figure 2. Relationship between fatigue limit and inclusion size.

low-strain/long-life region. Similarly, the statistical distribution of the recorded inclusion sizes for each process is considered. The distributions were found to follow the Gumbel distribution and to be different, confirming the initial assumption. Moreover, the size of extreme inclusions for the different materials is correlated to fatigue limits obtained in the experimental tests [4].

\section{Fatigue strength and defect size}

Considering crack growth properties taken from the literature [5], together with cyclic tests for the determination of cyclic elastic properties, it has been then possible to obtain the relationship between fatigue strength and inclusion size. This prediction successfully compares with the size of inclusions at fracture origin in the fatigue tests (Fig. 2). 
FDMDII - JIP 2014

\section{References}

[1] Adler, P. H., Allen, J. J. L., and Francis, R., J. ASTM Int. 4 (2007)

[2] Pelton, A. R., J. Mater. Eng. Perform. 20 (2011)

[3] Murakami, Y. et al., J. Test. Eval. 22 (1994)

[4] Urbano, M.F. et al, in Fatigue and Fracture of Medical Metallic Materials and Devices, ASTM STP 1559 (2013)

[5] Robertson, S.W. and Ritchie R.O., J. Biomed. Mater. Res., Part B: Appl. Bio- Mater. 84 (2007) 